\title{
Fourier analysis: the simplification of a complex waveform into simple component sine waves of different amplitudes and frequencies
}

\author{
Al Mamoojee iD \\ Department of Anaesthesia, Chris Hani Baragwanath Academic Hospital, University of the Witwatersrand, South Africa \\ Corresponding author, email: anisahmamoojee@gmail.com
}

\section{Summary}

Fourier analysis is the simplification of a complex waveform into simple component sine waves of different amplitudes and frequencies. ${ }^{1}$ A discussion on Fourier analysis necessitates reiteration of the physics of waves.

A wave is a series of repeating disturbances that propagate in space and time. ${ }^{2}$

- Frequency: the number of oscillations, ${ }^{2}$ or cycles per second. It is measured in Hertz and denoted as $1 /$ time or s$^{-1}$.

- Fundamental frequency: the lowest frequency wave in a series. It is also known as the first harmonic. Every other wave in the series is an exact multiple of the fundamental frequency. ${ }^{2}$

- Harmonic: whole number multiples of the fundamental frequency. ${ }^{1}$

- Amplitude: the maximum disturbance or displacement from zero caused by the wave. This is the height of the wave. ${ }^{2}$

- Period: time to complete one oscillation. ${ }^{2}$

- Wavelength: physical length of one complete cycle. ${ }^{2}$ This can be between two crests or two troughs. The higher the frequency, the shorter the wavelength.

- Velocity: frequency $x$ wavelength.

- Phase: displacement of one wave compared to another, described as $0^{\circ}-360^{\circ} .3$

A sine wave is a simple wave. It can be depicted as the path of a point travelling round a circle at a constant speed, defined by the equation ' $y=\sin x^{\prime} .{ }^{3}$ Combining sine waves of different frequency, amplitude and phase can yield any waveform, ${ }^{3}$ and, conversely, any wave can be simplified into its component sine waves.

Fourier analysis is a mathematical method of analysing a complex periodic waveform to find its constituent frequencies (as sine waves). Complex waveforms can be analysed, with very simple results. Usually, few sine and cosine waves combine to create reasonably accurate representations of most waves. Fourier analysis finds its anaesthetic applications in invasive blood pressure, electrocardiogram (ECG) and electroencephalogram (EEG) signals, which are all periodic waveforms. ${ }^{2}$ It enables monitors to display accurate representations of these biological waveforms.

Fourier analysis was developed by Joseph Fourier, a mathematician who analysed and altered periodic waveforms. ${ }^{2}$ It is done by computer programmes that plot the results of the analysis as a spectrum of frequencies with amplitude on the $y$-axis and frequency on the $x$-axis.

Keywords: Fourier analysis, complex waveform, simple component sine waves, amplitudes, frequencies

\section{Applications}

\section{Arterial pressure monitoring}

In the intra-arterial blood pressure monitoring system, complex waveforms are simplified by a microprocessor into their constituent sine waves, which are then reconstructed from the fundamental frequency, and eight or more harmonic waves of higher frequency to give an accurate depiction of the original wave. ${ }^{3}$ The greater the number of harmonics used to reconstruct the waveform, the more likely it is to be an accurate representation of it. ${ }^{4}$

To maintain accuracy, this system detects and transmits the high frequency components of the arterial waveform. ${ }^{3}$
The natural frequency is the frequency at which an object oscillates at freely when something causes it to move. ${ }^{1}$ If an object is subjected to a force with a similar frequency, it will oscillate at an increased amplitude. This is called resonance. ${ }^{1}$

If the natural frequency of an invasive blood pressure monitoring system is similar to the frequency of any sine wave components of the arterial waveform, the system will resonate, be excessively amplified, and as a result will have a distorted signal, manifested on the monitor as a wide pulse pressure and raised systolic blood pressure. $^{3}$

Although the heart rate is measured in beats per minute (bpm), for our purposes, it is beats per second (Hertz). The normal heart rate is $60-120 \mathrm{bpm}$ or $1-2 \mathrm{~Hz}$. The maximum heart rate that 
can be accurately monitored is around 180-240 bpm (3-4 Hz), seen clinically in a tachycardic neonate or in a supraventricular tachycardia. The heart rate is the fundamental frequency of the monitoring system. ${ }^{1}$

For accurate measurement of pressure, the catheter-tubingtransducer system must be capable of responding adequately to the highest frequency of the arterial waveform. The natural frequency of the measuring system must exceed the natural frequency of the arterial pulse which ranges from 16 to $24 \mathrm{~Hz} .^{3}$

In order for the measuring system to have a very high natural frequency, approximately 8-10 times the fundamental frequency of the arterial waveform (pulse rate), using a heart rate $180 \mathrm{bpm}$, we find that the natural frequency must be at least $8 \times 180 \mathrm{bpm}=$ $1440 / 60$ seconds $=24 \mathrm{~Hz}^{3}$

The natural frequency is determined by the properties of the components of the system. The natural frequency can be optimised by minimising the cannula/tubing length and compliance, lowering the density of the fluid used in the tubing, and making use of large diameter cannula/tubing. ${ }^{3}$

The intra-arterial monitoring systems we use in theatre have a natural frequency of $200 \mathrm{~Hz}$. The addition of three-way taps and long lengths of tubing reduces this. A similar effect occurs with air bubbles and blood clots in the system. To maximise the natural frequency, we make use of short, stiff, wide cannulae, which are $4 \mathrm{~cm}, 20 \mathrm{G}$ and non-tapered; that are connected to transducers that have a stiff, low-displacement diaphragm by non-compliant plastic tubing and a single 3-way stopcock. We also ensure there are no air bubbles, blood clots or kinks in the system. ${ }^{3}$

The natural frequency can be measured using the fast-flush test. The system is flushed with high-pressure heparinised saline, which creates an over and undershoot of pressure waves, resonating at the natural frequency of the measuring system. This frequency can be worked out using the formula: screen or paper speed/wavelength. A speed of $25 \mathrm{~mm} / \mathrm{s}$, and wavelength of $1 \mathrm{~mm}$, results in a natural frequency of $25 \mathrm{~Hz}{ }^{3}$

Anything that reduces the energy in an oscillating system is called damping, and, in contrast to resonance, this will underestimate the pulse pressure. ${ }^{3}$

\section{The electroencephalogram}

Depth of anaesthesia monitoring is an integral component of providing safe planes of anaesthesia and ensuring that awareness does not occur. With increased use of total intravenous techniques of anaesthesia, specifically target controlled infusion ( $\mathrm{TCl}$ ) models, an equivalent monitor to the minimum anaesthetic concentration (MAC) of volatile based anaesthesia had to be found. The bispectral index (BIS), and entropy are examples of such monitors, the physics of which are rooted in EEG analysis.

The EEG is a graph of brain electrical activity against time. Twenty electrodes on the scalp record the potential difference (voltage) between 16 combinations of these electrodes. ${ }^{1}$ EEG waves can vary between 50-200 microvolts, and have a maximum frequency of $100 \mathrm{~Hz}{ }^{1}$

The raw EEG can be processed by Fourier analysis into constituent sine waves. As the EEG comprises many different frequencies and amplitudes, most forms of analysis make use of specific time periods, or epochs, of the EEG, of 2-16 seconds in duration, and are displayed as time or frequency domain

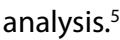

At deep planes of anaesthesia, low EEG frequencies are more common. Each cluster of frequencies is displayed on a compressed spectral array histogram, a method of displaying large amounts of analysed EEG data in an understandable format. ${ }^{1}$

From quantification of the frequency spectrum, multiple indices can be obtained, such as median power frequency, which is the value where $50 \%$ of the power spectrum is below and above that value; and spectral edge frequency, where $95 \%$ of the power spectrum is below that value.

As the spectral edge frequency is a marker of the highest occurring frequencies, this has been suggested to give an indication of the depth of anaesthesia. Its value drops as the plane of anaesthesia deepens, but it does not correlate well with drug concentration, particularly during the emergence phase of anaesthesia, ${ }^{1}$ compared to the median power frequency, which has an improved correlation with drug concentration, but the caveat is that its values are not consistent between patients and differ with different anaesthetic agents. ${ }^{1}$

\section{Conflict of interest}

The author declares no conflict of interest.

\section{Funding source}

None.

\section{ORCID}

Al Mamoojee (iD https://orcid.org/0000-0003-2334-3076

\section{References}

1. Aston D, Rivers A, Dharmadasa A. Equipment in anaesthesia and critical care: a complete guide for the FRCA. United Kingdom: Scion; 2014.

2. Middleton B, Philips J, Thomas R, Stacey S. Physics in anaesthesia. United Kingdom: Scion; 2012

3. Jones $\mathrm{A}$, Pratt $\mathrm{O}$. Physical principles of intra-arterial blood pressure measurement [Internet]. 2009 [cited 2020 Aug 9]. Available from: www.frca.co.uk.

4. Butterworth, JF, Mackey DC, Wasnick JD. Morgan \& Mikhail's clinical anesthesiology. 6th edition. McGraw Hill Lange; 2018.

5. Magee P. Physics for anaesthesia: magnetic resonance imaging; depth of anaesthesia monitoring; LASER; and light spectroscopy. BJA Educ. 2018;18:102-8. https://doi.org/10.1016/j.bjae.2017.12.004 